\title{
RESEÑA DE LIBRO - RAMOS VÁZQUEZ, ISABEL. LA FORMACIÓN DEL DERECHO OBRERO EN EL REINO UNIDO, FRANCIA Y ESPAÑA ANTES DE LA PRIMERA GUERRA MUNDIAL, UNIVERSIDAD DE JAÉN, THOMSON REUTERS ARANZADI, 2020. PRÓLOGO DE ANICETO MASFERRER
}

\author{
Maria Jesús Espuny Tomàs \\ Profesora Emérita de Historia del Derecho y de las Instituciones
}

Universitat Autònoma de Barcelona

Daniel Vallès Muñío

Profesor Lector Serra Húnter de Historia del Derecho y de las Instituciones Universitat Autònoma de Barcelona

\begin{abstract}
En este trabajo se resume y se reseña el libro de la Dra. Isabel RAMOS VÁZQUEZ titulado «La formación del derecho obrero en el Reino Unido, Francia y España antes de la Primera Guerra Mundial».

This work summarizes and reviews the book writes by Dr. Isabel RAMOS VÁZQUEZ entitled "The formation of labor law in the United Kingdom, France and Spain before the First World War."

Title: Book review. RAMos VÁZQUEZ, Isabel. La formación del derecho obrero en el Reino Unido, Francia y España antes de la Primera Guerra Mundial, Universidad de Jaén, Thomson Reuters Aranzadi, 2020. Prologue of Aniceto MASFERRER.

Palabras clave: derecho laboral, historia del derecho, derecho comparado, España, Francia, Reino Unido

Key words: labor law, legal history, comparative law, Spain, France, United Kingdom

IUSLabor 1/2021, ISSN 1699-2938, p. 338-350

DOI. 10.31009/IUSLabor.2021.i02.13
\end{abstract}

Fecha envío: 28.2.2021 | Fecha aceptación: 28.4.2021 


\section{Sumario}

1. La autora

2. La estructura y el contenido del libro

3. Los valores del libro 


\section{La autora}

Isabel RAMOS VÁZQUEZ, catedrática de Historia del Derecho y de las Instituciones en la Universidad de Jaén, cuenta con una larga trayectoria científica desarrollada en distintos ámbitos histórico-jurídicos, que acredita su capacidad de trabajo, la rigurosidad de sus investigaciones y su buen ejercicio con la pluma.

El libro que reseñamos estudia la formación del derecho obrero desde una perspectiva comparada. Como dice en el prólogo del Dr. Aniceto MASFERRER, la publicación es "una muestra ejemplar de cómo historiar el derecho desde" esa perspectiva. El análisis propuesto es de amplio espectro: tiene un alcance supranacional y va desde finales del siglo XVIII hasta la Primera Guerra Mundial.

Desde hace unas décadas, la Historia del Derecho y de las Instituciones ha ampliado su campo de investigación, fruto, sobre todo, de la aparición de nuevas titulaciones que exigen a sus docentes una mayor interdisciplinariedad. La transformación de la primigenia legislación del trabajo en el derecho del trabajo empezó a integrarse en los trabajos de una nueva generación de investigadores que son ejemplo de esa transversalidad y comparten su objeto de estudio con otras materias jurídicas y sociales. Así, la autora hace en el libro una doble demostración de sus conocimientos: tanto en derecho comparado como en la formación histórica del derecho social o derecho obrero, a la que se ha dedicado en muchos de sus trabajos. Lo segundo es condición de lo primero.

\section{La estructura y el contenido del libro}

Un magnífico prólogo de Aniceto MASFERRER encabeza la obra. En él se hace una referencia explícita y documentada sobre la actividad docente, investigadora y de gestión académica de la autora. Ello y su formación jurídica que le autorizan y justifican la empresa de este libro. El catedrático de la Universidad de Valencia valora la relación entre Historia del Derecho y derecho comparado y hace una rápida, aunque importante, reflexión sobre el descuido de la tradición española en los estudios de derecho comparado. Por ello, no tiene reparos en recomendar la traducción del libro al inglés para suplir ese descuido. Y nosotros, desde aquí, solo podemos que estar de acuerdo con el prologuista: traduzca el libro. Sería bueno para todos.

La obra está dividida en cinco capítulos precedidos por una "Introducción". La autora utiliza un marco cronológico que se inicia en una primera fase "nacional" de construcción del derecho obrero, en la que los diferentes Estados deciden su intervención para solucionar los problemas sociales derivados del liberalismo y de la 
industrialización. Una rápida referencia a las primeras Conferencias Internacionales o instituciones anteriores a la Primera Guerra Mundial, como la Asociación Legal para la Protección legal de los Trabajadores, considerada el precedente de la Organización Internacional del Trabajo. Es la creación de este organismo en el seno de la Sociedad de Naciones en 1919 con la que se inicia la "fase internacional", que cierra el marco cronológico del estudio. La "Introducción” justifica el doble objetivo de la metodología comparada: "comprender mejor el espíritu del desarrollo del derecho obrero español en un marco transnacional propio de la época en la que surgió". Además, propone un segundo objetivo: analizar el derecho obrero español dentro de la realidad europea y la ausencia del primero en las obras clásicas de la materia.

El primer capítulo titulado "Revolución liberal y trabajo" empieza con un epígrafe general en el que precisa el concepto de trabajo en el liberalismo económico y en el liberalismo jurídico. El análisis y las referencias a las obras de autores ingleses y franceses (Adam SMith, John Locke, Montesquieu o VoltAire) se completan con el estudio paralelo de las Constituciones francesas y de los Códigos napoleónicos. Pero no olvida a los autores españoles influenciados por el corporativismo de BENTHAM.

Se recoge una amplia bibliografía actualizada sobre el estado de la cuestión en el tema, imprescindible para interesados. Además, la autora demuestra una formación sólida en las principales doctrinas filosóficas que se iniciaron en el siglo XVIII y que conformaron los ordenamientos jurídicos de los primeros Estados liberales: pe. el concepto de un trabajo disociado del derecho de propiedad libre y de naturaleza individual en el marco de la contratación privada, o idolatrada la libertad del propietario para la contratación, al margen de las antiguas corporaciones y de las relaciones de servidumbre.

El segundo epígrafe de este primer capítulo trata del fin de la servidumbre y de la abolición del régimen corporativista o gremial, dentro de la liberalización de las relaciones de trabajo. La subdivisión no es baladí: en un primer bloque estudia la disolución del régimen de servidumbre en el campo, un ambicioso objetivo donde analiza con amplitud y precisión la experiencia francesa y su referencia para los legisladores gaditanos que antes incluso de la Constitución, se apresuraron a abolir los derechos jurisdiccionales de los señores y las relaciones de servidumbre, aunque mantuvieron y reforzaron el sagrado derecho de propiedad.

Junto a las numerosas citas bibliográficas, hemos de destacar las abundantes referencias sobre las disposiciones dictadas por las Cortes de Cádiz y sobre el restablecimiento de la abolición del régimen señorial al advenimiento de Fernando VII. De forma breve pero no exenta de interés, la autora dedica su atención al origen de la figura del "cacique" y a 
los jornaleros agrícolas captados por el movimiento social que más tarde se conocería como "movimiento obrero": una reflexión imprescindible para entender el devenir de las relaciones laborales del siglo XIX. He aquí uno de los activos de la obra: invita al lector atento a preguntarse sobre materias en las que la ortodoxia académica cree que todo ha sido escrito.

La abolición del régimen corporativista de artes y oficios en Francia forma parte de la segunda subdivisión. Es relevante el excelente uso que RAMOS VÁZQUEZ hace de la obra del Ministro de Finanzas de Luis XVI, Turgot, que conoce con profundidad, y de otras fuentes normativas imprescindibles para este período. Es interesante el estudio del delito de coalición (coligación, en España) en el par de aspectos que podía revestir: el control del asociacionismo obrero y la codificación penal francesa. Le sigue un análisis detallado sobre el primigenio derecho de asociación obrera en Francia y una de las consecuencias de su regulación, en la primera mitad del siglo XIX: la proliferación al margen de la legalidad de asociaciones de trabajadores, clubes políticos o sociedades secretas.

La efímera abolición del régimen gremial en el Reino Unido integra la tercera parte de esta subdivisión. Resulta muy interesante el desarrollo que la autora hace de la extensión a este país de la persecución y el castigo de cualquier tipo de asociación de patronos o de trabajadores relacionada directamente con la libertad de trabajo. Recuerda que la reivindicación de mejoras salariales y mejores condiciones laborales se inició a finales del siglo XVIII, si bien las actuaciones políticas y las acciones legales proliferaron a partir de la actuación del primer Ministro WiLliam PITT y de las Combination Act de 1799 y de 1800, que penalizaban las asociaciones.

El análisis exhaustivo de las dos normas está acompañado por una extensa bibliografía y parte del texto de la última ley. La campaña para derogar las Combination Act de 1799 y de 1800 está ampliamente desarrollada, siguiendo, en paralelo, las actuaciones de los trabajadores, las formas de represión de los patronos y el movimiento protagonizado por políticos y juristas, contrarios a la represión del asociacionismo. Por ello, el Reino Unido se convertiría en un líder del movimiento sindicalista en toda Europa.

Finalmente, la cuarta parte se dedica a la abolición de los gremios en España. La fórmula que utiliza la Dra. RAMOS VÁZQUEZ renueva el relato de un episodio conocido. Probablemente la reproducción de una parte del Informe de Jovellanos de 1758 resulta un elemento imprescindible para valorar la situación anterior al conocido Decreto del Conde de Toreno. Las vicisitudes surgidas al restablecimiento del Absolutismo al retorno de Fernando VIII se analizan hasta la definitiva abolición de los gremios en 1836. La normativa de los diferentes Códigos Penales de 1822 y 1848 se mezcla con los 
movimientos obreros organizados que se producen con el ludismo en Alcoy, en Camprodón o en la Fábrica Bonaplata. No olvida a las Sociedades de Socorros Mutuos y los avatares surgidos por la desconfianza que producían sus actividades dentro de una encubierta legalidad. La narración de los hechos relacionados con el asociacionismo obrero es absolutamente necesaria para comprender un momento histórico y una esperanzadora propuesta jurídica como el Proyecto de Ley sobre la Industria Manufacturera de Alonso Martínez en 1855 que no llegó a hacerse realidad.

La conquista de los derechos de asociación y de huelga en Francia y en España constituye el tercer apartado del capítulo primero. Están subdivididos en dos bloques, uno que se inicia con la Ley Ollivier de 1864 y transcurre hasta las Leyes WaldeckRousseu (1884-1901) y el otro que va desde el Decreto de 1868 a las Leyes de Maura (1908-1909). Se trata de un auténtico trabajo de derecho comparado entre los dos países, sus leyes, sus Constituciones y sus Códigos Penales, el paso de la prohibición absoluta a la tolerancia, para terminar con la legalización a través de la derogación de las disposiciones que penalizaban el asociacionismo: en el espejo comparativo encontramos la Loi du 1er de julliet 1901 en Francia, que derogaba los artículos 291293 del Código Penal francés, y la Loi du 9 décembre 1905 para la derogación del artículo 294 del Código Penal, cuya imagen compara con la Ley Maura de 27 de abril de 1909, que derogó el artículo 556 del Código Penal español.

El capítulo segundo se dedica a "La contratación privada del trabajo y las bases ideológicas del derecho obrero". Siguiendo la sistemática utilizada, el capítulo se subdivide en diferentes epígrafes sobre los países que son objeto de este trabajo. Sin duda, ello ayuda a la comprensión del ejercicio comparativo.

En primer lugar, trata la contratación privada del trabajo y las obligaciones de las partes. A partir de ahí, aparecen dos bloques diferenciados, pero absolutamente relacionados entre sí: el primero, se inicia con un estudio del contrato de servicio (contract of service) en el Reino Unido, con especial referencia a la Master and Servant Act de 1823; el segundo, explica el arrendamiento de obra e industria (louage d'ouvrage et d'industrie) en Francia y finaliza con el "arrendamiento de obras y servicios" en España.

La escasez de regulación del arrendamiento de servicios en los Códigos civiles francés y español impulsa a la autora a un extraordinario epígrafe en el que estudia las obligaciones de las partes en la doctrina de los dos países: es unos de los temas capitales para el estudio del origen del derecho obrero, tratado desde la doctrina de derecho público o del derecho privado. Termina con una particular manifestación francesa: el livret d'ouvrier, la cartilla obrera, un documento que no se regula en Reino Unido, ni en 
España. La expresión de las semejanzas y de las diferencias entre ordenamientos jurídicos son esencia de la comparación.

El propio enunciado de la segunda parte de este capítulo contiene un proyecto futuro: hacia una doctrina jurídico social, es decir, hacia las bases ideológicas del derecho obrero, dando desde el inicio la connotación de evolución de viaje intelectual. El primer epígrafe se dedica a las "las primeras voces críticas contra el sistema liberal" y es, posiblemente, el estudio más completo y bien estructurado en derecho comparado sobre el tema.

Se trata de un brillante ejercicio, donde junto a los movimientos obreros, se señala a los pioneros del socialismo utópico como Robert Owen o Fourier, Cabet o Saint Simon en Francia y los españoles con sus influencias internacionales. Los políticos reformistas ingleses, siguiendo las corrientes utilitaristas y humanitarias de sus antecesores, lograron ir educando a la opinión pública sobre la necesidad de implementar mejoras sociales. Es interesante la mención que hace de las mujeres que, manifestando una inquietud social, escribieron sobre el problema obrero: Ellen Smith (hija de John Stuart Mill), Victorine JEANS, Isabel TAYLOR y Beatrice WEBB, quien junto a su marido Sidney WebB fundó la Sociedad Fabiana a la que pertenecía Emmeline PANKHURST, entre otros miembros ilustres.

Sobre Francia, la autora recoge los autores más representativos de la economía, destacando la personalidad de Jules Simon, autor intervencionista partidario de la legislación protectora del trabajo infantil y femenino. Señala los autores que, desde el campo de la economía social, recuperan las propuestas de una economía solidaria y un derecho social. La legislación obrera y el corporativismo cuentan también con importantes representantes que, en el caso de Albert de Mun y el baron Piérard, lo son desde el catolicismo social. La Dra. RAMos VÁZQUEZ no deja de comentar los trabajos para una Conferencia Internacional para la Protección Legal de los Trabajadores, donde destacó, junto a otros importantes juristas, la figura del profesor de Derecho, Raoul Jay, cuyas obras fueron traducidas en España por Adolfo PosADA.

La defensa del intervencionismo del Estado frente a la cuestión social contó en España con la defensa que de ello hicieron los socialistas, los krausistas, los conservadores como Cánovas del Castillo o Dato y la influencia del catolicismo social desde la publicación de la Encíclica de Leon XIII, Rerum Novarum. Todo ello se consolidó con la llegada al poder de acreditados políticos que profesaban la doctrina krausista y que llevaron a cabo la reforma social en nuestro país a través de dos instituciones, la Comisión de Reformas Sociales, creada por Segismundo MORET en 1883 i el Instituto de Reformas Sociales, por Gumersindo DE AZCÁRATE en 1903. 
La protección de los menores y de las mujeres dentro de la primera legislación obrera de carácter excepcional es el tema al que dedica el capítulo tercero, que se divide a su vez en dos bloques. Uno, dedicado a la salvaguarda de los menores en el trabajo industrial y el otro, a la incorporación de las mujeres a la legislación laboral.

Si concretamos, el primero explica el trabajo de los niños en el Reino Unido y en Francia, para continuar con la legislación española. El interés básico era que los niños se formaran en el aprendizaje de un oficio, por lo que su productividad sería en beneficio del Estado. Este aspecto es analizado por la autora aportando la normativa de cada momento histórico reguladora del trabajo infantil, a la vez que examina las corrientes filantrópicas, normalmente avaladas por médicos y sociedades humanitarias, que estudiaban las consecuencias del desgaste físico y las duras condiciones laborales en la salud de los pequeños.

Las leyes británicas y francesas incidían en los mismos aspectos, horarios, descanso para la comida, potenciar la educación y, especialmente, crear un servicio de inspección de los puestos de trabajo ocupados por niños y jóvenes. La investigación llevada a cabo por la Dra. RAMOS VÁZQUEZ permite seguir puntualmente el desarrollo de la legislación sin descuidar la acción de los políticos y de los informes médicos, como los de Villermé o Dupin en Francia. A saber: no solo se explican las normas, sino que se las pone en contexto, ampliando el relato necesario para la historia del derecho. Y se agradece, mucho.

El segundo aspecto de la protección de menores desgrana la ampliación y diversificación de la protección jurídica de los menores, partiendo de las leyes de fábricas inglesas de 1850 y 1853 que completaban la legislación anterior. En una situación similar, las leyes francesas convergieron en el futuro Code du travail et de la prévoyance social. Pero la explicación de la legislación española añade la comparación que la autora realiza con las normas británicas y francesas, ya conocidas. Para concluir que los países analizados coinciden en la prohibición del trabajo infantil nocturno y el descanso dominical obligatorio, que se integran rápidamente a la diferente concreción de las horas laborables, la educación, los certificados de salud y las prohibiciones de trabajos peligrosos existentes en todos los países.

La mujer será considerada como menor, a efectos de su capacidad y de su "media fuerza" laboral, y las consecuencias de esta categorización se sucedieron a partir de la Revolución Industrial. 
La preocupación de que la mujer fuera una competidora del hombre y abandonara las tareas domésticas y de cuidado familiar estaba presente en la legislación que la Dra. RAMOS VÁZQUEZ estudia, valora y compara en un esfuerzo integrador.

El segundo epígrafe está dedicado a la protección a la maternidad mediante el descanso puerperal. Resulta muy innovador la relación que la autora hace del movimiento asociacionista femenino en defensa de la maternidad (maternalistas británicas y francesas), impulsado o no por el feminismo, en los países objeto de su estudio y su influencia en la legislación de la norma española, la Ley Dato de 13 de marzo de 1900, que fue pionera.

La situación de los derechos de maternidad para las mujeres trabajadoras antes de la conferencia de Washington de la OIT (1919) en Reino Unido, Francia y España queda absolutamente clarificada. Un epígrafe final, breve, que siempre es mejor, recoge otros derechos específicos de la mujer obrera con la perspectiva comparada: la libre disposición del salario de la mujer casada, la exclusión de los sindicatos, la prohibición de algunos trabajos de la mujer, la ley de la silla o la prohibición del trabajo nocturno para la mujer forman parte de éste.

La generalización de la protección al trabajador adulto integra el capítulo cuarto. Los aspectos a los que dedica los diferentes apartados se hallan absolutamente conectados entre sí. En primer lugar, trata la higiene y la seguridad en el trabajo; a continuación, los accidentes de trabajo y las enfermedades profesionales. Un planteamiento preliminar indica que se exponen únicamente las primeras medidas preventivas en los tres países, unas disposiciones en las que hubo una influencia directa por parte de los médicos higienistas, que con sus estudios llamaron la atención de las condiciones malsanas del trabajo industrial.

El examen de la higiene y la seguridad en el Reino Unido se inicia necesariamente por la Factory Act de 1844, a la que siguieron otras normas reguladoras del uso de sustancias insalubres y peligrosas que limitaron el trabajo de las mujeres y de los menores en algunos centros laborales. También señala la ley de fábrica o Factory and workshop Act de 1878 como un compendio de la normativa anterior. Se analiza con detalle el contenido y también la acción de los inspectores, elementos indispensables para optimizar los resultados en el cumplimiento de la ley. Es muy interesante la preocupación del control de la salud de los trabajadores externos o "a domicilio", que obligaba a los propietarios a elaborar listas para que pudieran ser inspeccionados igual que sus talleres domésticos. 
Las primeras leyes francesas de higiene y de seguridad de los trabajadores parten de la misma premisa de la legislación británica: las normas originarias protegieron primeramente a mujeres y niños. La autora llega a la conclusión que el mérito fue abrir el debate sobre la responsabilidad del empresario o propietario en el caso de los accidentes de trabajo, un punto que ya se había empezado a tratar en el Reino Unido y al que dedica otro de los epígrafes de este capítulo.

La autora trata a continuación el caso español con la influencia de médicos y de higienistas. Además, su análisis permite un seguimiento completo de la legislación española sobre higiene y seguridad.

Los accidentes de trabajo y las enfermedades profesionales integran la segunda parte del capítulo cuarto. La autora desarrolla un estudio minucioso que va desde la teoría contractual a la teoría de la culpa, pasando por la teoría del riesgo profesional: la doctrina de la culpa aquiliana comparte espacio e interés junto a la doctrine of common employement del Reino Unido y Escocia, y con la théorie contractuelle de la jurisprudencia francesa que sigue la española. Como es sabido, en España no existió apenas transición entre la teoría contractual y de la culpa, y la teoría del riesgo profesional reconocida por la Ley de accidentes de trabajo de 30 de enero de 1900. Uno de los aspectos más significativos de esta parte es sin duda la valoración que la Dra. RAMOS VÁZQUEZ hace de otros países como Alemania o Dinamarca ampliando el ámbito de su investigación. Nos permitimos sugerir que no olvide el análisis comparativo de esos países en futuros trabajos: podría llegar a conformar un estudio europeo de la materia.

Las leyes de compensación o Compensation Acts británicas de 1897 y 1906 se examinan con sus precedentes, sus aportaciones y la jurisprudencia anterior y posterior, como un amplio ejercicio de derecho comparado. Y no solo eso: también se muestran las diferencias con el sistema de Bismark.

La teoría del riesgo francesa fue asumida por la Loi du 9 avril 1898, Concernant les responsabilités des accidents dont les ouvriers sot victimes dans leur travail, que deroga la anterior teoría del contrato común y la responsabilidad por culpa en el caso del trabajo. Se estudia la aplicación y la consolidación de esta ley, a pesar de que las enfermedades profesionales estarían excluidas hasta 1919.

La Ley de accidentes de trabajo española de 30 de enero de 1900 inicia un nuevo epígrafe del capítulo 4. Sus precedentes inmediatos y el reglamento de 28 de julio de 1900, que la desarrolló, se documentan a través de múltiples aportaciones. Un tema interesante es la enfermedad profesional y la solución jurisprudencial en relación este 
aspecto. La reforma de la primera Ley de accidentes de trabajo española siempre estuvo en el punto de mira del legislador, pero no llegó a producirse hasta después de la Primera Guerra Mundial en 1922.

Finalmente, se explican la expansión de los seguros sociales y la incorporación de riesgos no profesionales, dos elementos que cierran este capítulo. En primer lugar, se escribe sobre el tema de las aseguradoras, que fueron voluntarias en un principio en nuestra legislación y con un fondo de garantía en el caso francés. El sistema más avanzado fue el del Reino Unido, que la autora comenta a través de las diferentes normas y describe su funcionamiento hasta 1946, cuando la National Insurance Act abolió definitivamente las leyes de compensación y las reemplazó por un sistema de seguridad social con responsabilidad de todos los actores, incluso el propio Estado.

La represión y el castigo del asociacionismo obrero fue el motivo del escaso desarrollo del mutualismo en Francia y en España. La autora presenta la Loi du 5 avril 1910 que sigue el ejemplo alemán y el inglés. En nuestro país, se detalla la creación del Instituto Nacional de Previsión, con la finalidad de fomentar la previsión social y generalizar y gestionar las pensiones de retiro obrero. La Conferencia técnico-social para la implantación de los seguros sociales de 1917 supuso una ventaja importante en la gestión futura de los seguros sociales obligatorios.

El último capítulo se dedica a la mejora de las condiciones de trabajo y la evolución hacia el contrato de trabajo. Comienza con una presentación que lleva el título genérico de la protección de los salarios, la cual tiene dos bloques diferenciados: (1) la prohibición del truck system y del embargo de salarios, y (2) la protección del salario por el trabajo hecho "a destajo" o "en domicilio" y la fijación del salario mínimo en determinadas industrias.

El último de los aspectos retoma la explicación de las teorías económicas liberales y la regulación inicial del contrato de servicio, el arrendamiento de obra e industria y el arrendamiento de obras y servicios, con una dedicación generosa al Reino Unido, "el caso más avanzado en cuanto a la tasación de salarios". De Francia se destaca los Conseils de travail y de la legislación española, que no llega a formar organismos mixtos como en otros países que fijen ese salario mínimo.

La segunda parte del capítulo quinto trata de la jornada laboral y del descanso dominical. Los detalles que más llaman la atención: del Reino Unido "el largo camino por la limitación de la jornada laboral y el descanso dominical"; respecto a Francia "el triunfo de la protección de la jornada de trabajo y el descanso semanal "repos hebdomadaire"; y para España "la (consecución de) limitación de la jornada laboral y el 
descanso dominical". Queda patente el dominio de la doctrina y de las fuentes de los diferentes países por parte de la Dra. Isabel RAMOs VÁZQUEZ, que cumple plenamente los objetivos fijados.

Finalmente, a modo de epílogo final, explica el desarrollo desde el contract of service hacia el contract of employemont, y plantea los nuevos elementos de la redefinición de las relaciones laborales y la aparición de un modelo contractual. A continuación, el nacimiento del contrato de trabajo, contrat de travail en Francia y finalmente explica el impulso del "contrato de trabajo" en España.

\section{Los valores del libro}

Estamos ante un auténtico tratado de derecho comparado, una contribución fundamental que puede valorarse desde una perspectiva interdisciplinar: historia del derecho y de las instituciones, derecho del trabajo, derecho constitucional o incluso filosofía del derecho.

Los objetivos que la autora declara en la Introducción se superan con creces. Un ambicioso proyecto fruto de un trabajo constante y de una dedicación consciente a un tema complejo, que la Dra. RAMos VÁZQuEZ prueba domina. Resultaría difícil de señalar si ha existido predilección por alguno de los tres países objeto del libro: los autores, las disposiciones, las teorías, las escuelas se encuentran de forma pormenorizada, pero de manera equilibrada.

Un detalle no menor: en solo 348 páginas (bibliografía incluida) se da cuenta de todo lo que se ha resumido en los párrafos anteriores. Es un libro eficiente, sobre todo con el tiempo del lector. En poco espacio se explica todo lo que la autora quiere, sin dejarse nada en el tintero; en poco tiempo, el lector se queda con una visión comparada excelente del nacimiento del derecho del trabajo en tres países europeos. En los tiempos que corren, conseguir esa eficiencia en un libro de historia del derecho bien se merece un elogio. Y si, además, está muy bien escrito, poco podemos pedir más.

Tenemos que agradecer a la autora una visión preferente a la perspectiva de género. La presencia de mujeres cuya inquietud social, con o sin influencias familiares, llegaron a escribir sobre el problema obrero y protagonizaron importantes reformas en una sociedad empobrecida. La proliferación de movimientos asociacionistas femeninos o las maternalistas francesas e inglesas que enumera es significativa.

El libro describe con profundidad todas las cuestiones que puedan plantearse en el estudio de la formación del derecho obrero con la perspectiva comparada. La bibliografía consultada, que aparece en las notas a pie de página y se recoge in extenso 
en las páginas finales, supone un excelente expositor de las obras de los autores que histórica o actualmente son referencia inexcusable.

Otro pequeño detalle que es preciso resaltar: cada uno de los capítulos se halla encabezado por un párrafo relacionado con el propósito a desarrollar y que corresponde a un autor o autora de la literatura universal coetáneo con los inicios de la formación del derecho obrero. Así Charles Dickens (capítulo 1), Émile Zola (capítulo 2), Emilia Pardo Bazán (capítulo 3), Leopoldo Alas "Clarín" (capítulo 4) y Concepción Arenal (capítulo 5). Un guiño al lector: es posible (aun preceptivo) ampliar el campo de juego de la historia del derecho. Y la literatura está ahí. Debe estarlo.

La Dra. RAMOS VÁzQUEZ, a partir de su incursión como docente e investigadora en la historia social del derecho, ha superado con esta obra sus otros trabajos, para demostrar una auténtica maestría en el tema, no sólo como jurista sino también como historiadora. Pero, no se queden solo con ello: en el libro se insinúan otros temas para futuros trabajos. Los esperamos. 